\title{
Linear Array Synthesis for Wireless Power Transmission Based on Brain Storm Optimization Algorithm
}

\author{
Guang Xu Liu, Qin Qin, and Qing He Zhang (iD \\ The College of Computer and Information, China Three Gorges University, Yichang, China \\ Correspondence should be addressed to Qing He Zhang; zqh@ctgu.edu.cn
}

Received 16 October 2020; Revised 28 December 2020; Accepted 16 January 2021; Published 4 February 2021

Academic Editor: Andrea Francesco Morabito

Copyright (C) 2021 Guang Xu Liu et al. This is an open access article distributed under the Creative Commons Attribution License, which permits unrestricted use, distribution, and reproduction in any medium, provided the original work is properly cited.

Based on the brain storm optimization algorithm, this paper proposed a new method to optimize the beam collection efficiency of the linear antenna array. In the process of optimization, constraints such as aperture size and minimum antenna spacing are considered. In this paper, the optimization with different antenna apertures, different antenna angles, and different array numbers are studied. A series of representative data and simulation results are given and the superiority of the brainstorming algorithm is demonstrated by comparing with the genetic algorithm.

\section{Introduction}

Wireless power transmission (WPT) is a technology that transmits power wirelessly $[1,2]$. With the deepening of research, this technology has not only been applied to hightech fields such as solar power satellites but also reflected on various fields of daily life, charging mobile phones, computers, or electric cars $[3,4]$. In recent years, people are studying this technology from more and more aspects, hoping to make a breakthrough in different directions. For a WPT system, its main components include two parts: a transmitting antenna for transmitting energy towards the receiving region and a receiving antenna for converting and receiving energy. Therefore, people's research directions can be broadly divided into these two categories. For either of the two parts, the purpose is to achieve higher transmitting/ receiving efficiency.

In order to obtain higher power efficiency, people began to pay more attention to the research of transmitting antenna and proposed a series of methods, including the edge tapering method and stochastic optimization method $[5,6]$. In 2013, Oliveri et al. took BCE (beam collection efficiency, the ratio of the energy received to the total energy sent by the transmitting antenna) as the objective function and studied the two-dimensional planar antenna array by solving the generalized eigenvalues [7]. Then, more and more people have applied optimization algorithms, such as particle swarm optimization (PSO) algorithm and genetic algorithm (GA), to this field [8]. The abovementioned methods all have some disadvantages, such as complex implementation, severe parameter influence, and low precision. Therefore, many scholars devote themselves to the study of algorithms to get better performance.

Inspired by the creative problem-solving process of human beings, Professor Shi proposed the brain storm optimization (BSO) algorithm [9] in 2011. This algorithm uses the clustering idea to search the local optimal and obtains the global optimal by comparing the local optimal. The idea of variation increases the multiform of the algorithm, avoids the algorithm falling into the local optimal, and searches for the optimal solution in the process of polymerization and dispersion, which is novel and suitable for solving the problem of multipeak and high-dimensional functions. In [10], Rahmat-Samii used BSO to optimize electromagnetic devices' characteristics. The practicability and effectiveness of BSO algorithm in electromagnetic field are proved. Through reviewing the literature, we find that a lot of researches have been done on linear antenna arrays [11-14]. But to the best of the author's knowledge, no one has applied BSO to BCE optimizations with multiple constraints on linear antenna arrays, and the characteristics of BSO algorithm are very suitable for application in this field. 
Therefore, the purpose of this paper is to study the optimization of BCE with the BSO algorithm based on multiconstraint conditions.

In this paper, with the constraints of array number, aperture, and minimum array spacing, BCE is optimized by using uniform excitation nonequal spaced linear array and BSO algorithm. The outline of the paper is as follows. The optimized problem is mathematically formulated in Section 2. A brief introduction to the $\mathrm{BSO}$ algorithm is given in Section 3. Numerical simulation and analysis are described in Section 4. Finally, some conclusions are drawn in Section 5.

\section{Materials and Methods}

Figure 1 shows the geometry of the transmitting part of the linear antenna array. As we can see from Figure 1, the transmitting antenna is composed of $2 \mathrm{~N}+1$ antenna units (the analysis of $2 \mathrm{~N}$ antenna units is the same as that of $2 N+1)$; its array factor can be expressed as follows:

$$
E(u)=\sum_{n=-N}^{N} I_{n} \exp \left(j k u d_{n}\right)=I_{0}+\sum_{n=0}^{N} 2 I_{n} \cos \left(k u d_{n}\right),
$$

where the $d_{n}$ and $I_{n}(n=-N, \ldots, N)$ represent the element coordinate and current; let $d_{N}=L$ and $d_{-N}=-L$, thus the aperture size is always $2 L ; u=\cos (\theta), \theta$ represents the steering angle from the broadside of the array, $0 \leq \theta \leq \pi ; k=2 \pi / \lambda$ being the wavenumber, $\lambda$ represents the wavelength, respectively. In this paper, all the element currents are supposed to be uniform, that is, assume $I_{n}=1$, for all $n$ (if transmitting antenna is composed of $2 \mathrm{~N}$ elements, $I_{0}$ does not exist), and the corresponding power pattern can be formulated as

$$
P(u)=|E(u)|^{2}=\left[I_{0}+\sum_{n=0}^{N} 2 I_{n} \cos \left(k u d_{n}\right)\right]^{2} .
$$

The definition mentioned above of BCE is the ratio of the energy received by the receiving antenna to the total energy sent by the transmitting antenna. Therefore, BCE can be expressed as

$$
B C E=\frac{P_{\psi}}{P_{\Omega}}=\frac{\int_{\psi} P(u) \mathrm{d} u}{\int_{\Omega} P(u) \mathrm{d} u},
$$

where $\psi$ and $\Omega$ are the angular region identifying the radiated area and the whole visible range, respectively.

Taking BCE as the objective function, the optimization process can be expressed as searching the position vector of antenna elements $d=\left(d_{0}, d_{1}, \ldots, d_{N}\right)^{T}$ within a certain aperture size to achieve the maximum BCE, and $\left|d_{i}-d_{j}\right| \geq d_{c}$, viz.

$$
\left\{\begin{array}{l}
\min f(d)=-B C E(d), \\
\text { subject to } l_{0} \leq d_{n} \leq L, \quad n=\{0,1, \ldots, N\}, \\
d_{i}-d_{j} \geq d_{c}, \quad 0 \leq j<i \leq N,
\end{array}\right.
$$

where $l_{0}$ represents the distance from the first antenna element to the $Y$ axis. The objective function $f(d)$ equals BCE,

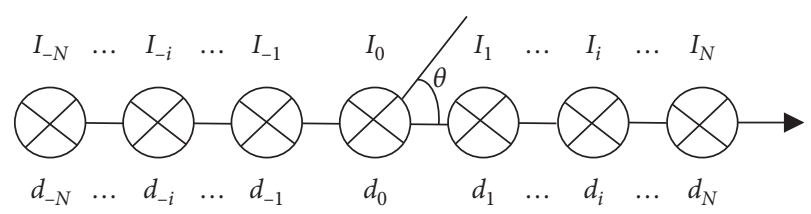

Figure 1: Geometry of the nonuniformly linear symmetric array.

and the negative sign is used to make it a minimization problem. It is easy to prove that by using the method in [15], all the optimal variables can meet the constraints.

\section{Brain Storm Optimization Algorithm}

As mentioned above, BSO uses the clustering idea to search the local optimal and obtains the global optimal by comparing the local optimal. Its pseudocode is given in Figure 2 and BSO algorithm can be described as follows:

(1) Population initialization: in this step, a population $X$ is randomly generated. The population's size is $N$ and the problem dimension is $D$. So, this population can be described as

$$
X^{i}=\left[x_{1}^{i}, x_{2}^{i}, \ldots, x_{D}^{i}\right], \quad 1 \leq i \leq N .
$$

For every $x^{i}$, it can be randomly generated as

$$
x^{i}=x_{\min }+\operatorname{rand} *\left(x_{\max }-x_{\min }\right),
$$

where $\left(x_{\min }, x_{\max }\right)$ are the specified minimum and maximum values for each dimension. The random number function rand returns a number between 0 and 1 .

(2) Cluster: BSO uses a $k$-mean clustering method to divide the $N$ ideas into $M$ clusters.

(3) Rank: before this step, evaluate each idea with the objective function. Rank each idea according to the evaluation results, and then select the best idea in each group as the cluster center.

(4) Disrupting cluster center: to avoid deception and increase population diversity, we occasionally replace a cluster center with a randomly generated idea. The disrupting operator of $\mathrm{BSO}$ is given as lines 6-8 in Figure 2.

(5) Update: the first step is to determine whether a new idea is based on one cluster or two clusters. This step depends on the parameter p_one. The updating operator of BSO is given as lines 9 to 26 in Figure 2. If the new idea is based on one cluster, it can be described as follows:

$$
x_{\text {new }}=x_{d}+\xi * N(\mu, \sigma) \text {, }
$$

where $x_{d}$ is the selected idea, $N(\mu, \sigma)$ is the Gaussian random value with mean $\mu$ and variance $\sigma$, and $\xi$ is a coefficient that weights the contribution of the Gaussian random value and can be described as follows: 


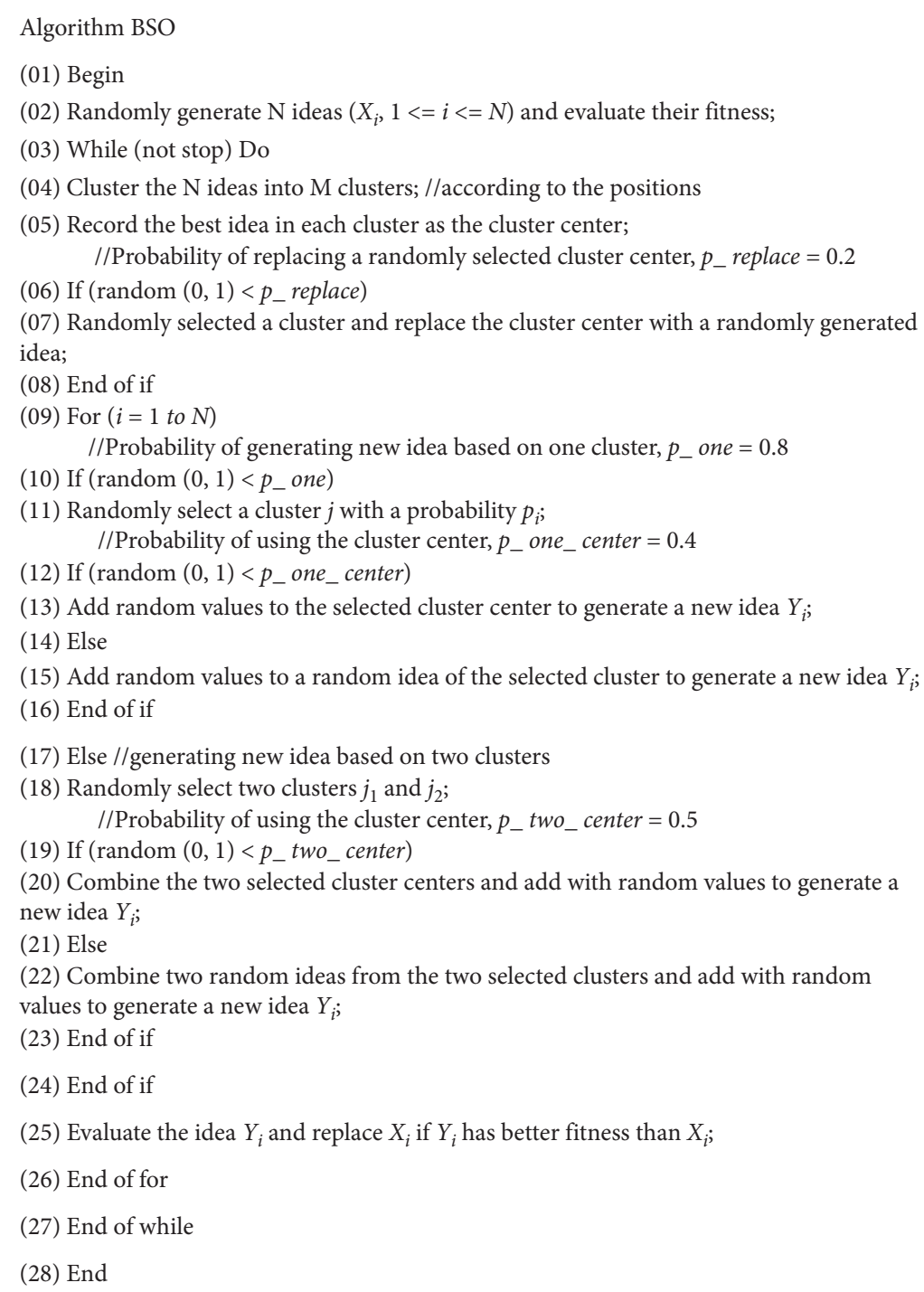

FIGURE 2: Pseudocode of brainstorm optimization algorithm.

$$
\xi=\operatorname{logsig}\left(\frac{(0.5 * G-g)}{k}\right) \times \operatorname{random}(0,1),
$$

where logsig () is a logarithmic sigmoid transfer function, $G$ is the maximum number of iterations, $g$ is the current iteration number, $k$ is for changing logsig () function's slope, and rand () is a random value within $(0,1)$. If the new idea is based on two clusters, the idea $x_{d}$ is calculated as follows:

$$
x_{d}=R * x_{d 1}+[1-R] * x_{d 2},
$$

where $R$ is a random value within $(0,1)$, while $x_{d 1}$ and $x_{d 2}$ are the two ideas from clusters $j_{1}$ and $j_{2}$, respectively. Then, use (7) for the update operation.

\section{Results and Discussion}

The purpose of this section is two-fold: on the one hand, to test the effectiveness of BSO according to computational efficiency and flexibility when dealing with different aperture sizes and different receiving areas. On the other hand, to compare the results obtained through the BSO method to that through the GA method. Towards this end, a set of simulations have been carried out by assuming. For the BSO algorithm, the parameters we selected are as follows: p_replace $=0.2, \quad p \_$one $=0.8, \quad p \_$one_center $=0.4$, and p_two_center $=0.5$, and it will be used with five clusters by utilizing the k-mean clustering algorithm. The crossover and mutation probabilities for GA are set to $P_{c}=0.7$ and $P_{m}=0.01$, respectively. Both algorithms will have the same population of $N=30$; also, the maximum number of iterations is set to 500. Both algorithms are executed 20 times. Considering the efficiency of the algorithm, we take the minimum adjacent element spacing $d_{c}$ as the fixed population starting position for each run.

The first set of experiments was conducted in the case of $2 N=10$ elements. In this set of experiments, the parameters involved in (4) are set as $d_{c}=0.4 \lambda$ and $l_{0}=0.5 d_{c}$. In order to 
TABLE 1: Maximum solutions in BSO, $2 N=10$, normalized to $0.4 \lambda$.

\begin{tabular}{|c|c|c|c|c|c|c|}
\hline$u_{0}$ & \multicolumn{6}{|c|}{ Optimized $d$ vector } \\
\hline 0.1 & $d=$ & $(0.615$ & 1.658 & 2.927 & 4.116 & $5.625)$ \\
\hline 0.2 & $d=$ & (0.517 & 1.517 & 2.613 & 3.945 & $5.625)$ \\
\hline 0.4 & $d=$ & $(0.803$ & 2.088 & 3.232 & 4.232 & $5.625)$ \\
\hline
\end{tabular}

TABLE 2: Maximum and average BCE in BSO and GA.

\begin{tabular}{lcccccr}
\hline$u_{0}$ & & 0.1 & & & 0.2 & 0.4 \\
BCE $(\%)$ & Max. & & Avg. & Max. & Avg. & Max. \\
\hline GA & 79.17 & & 79.09 & 95.80 & 95.62 & 98.32 \\
BSO & $\mathbf{7 9 . 1 9}$ & & $\mathbf{7 9 . 1 9}$ & $\mathbf{9 5 . 8 1}$ & $\mathbf{9 5 . 8 1}$ & $\mathbf{9 8 . 3 5}$ \\
Comparison & 0.02 & & 0.1 & 0.01 & 0.19 & $\mathbf{9 8 . 3 5}$ \\
\hline
\end{tabular}

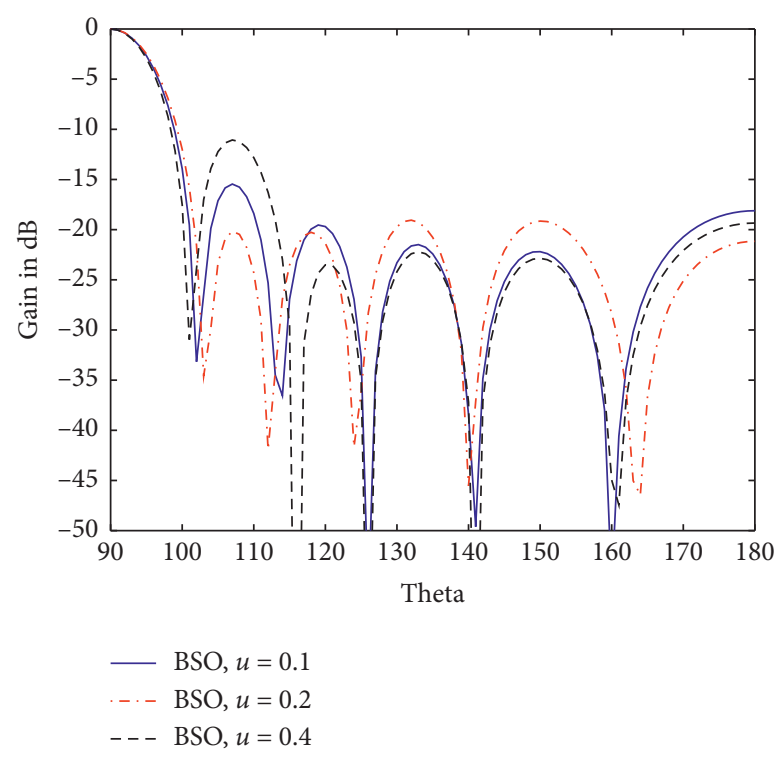

(a)

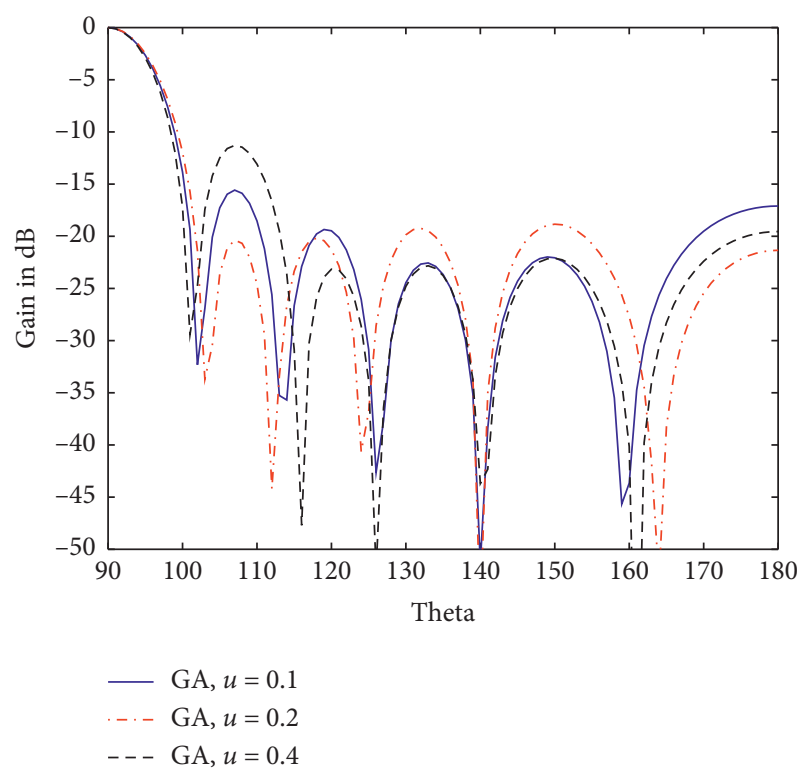

(b)

FIGURE 3: Different $u_{0}$ examples: best-normalized power pattern found by BSO (a) and GA (b) (in 20 times runs).

make the spacing of all adjacent antenna elements meet $d_{c}$, the aperture size is set as $2 L=4.5 \lambda$, that is, the average element spacing is $0.5 \lambda$, and we changed the size of the receiving region $\psi$ by changing $u_{0}$. In this case, the BCE can be formulated as follows:

$$
B C E=\frac{P_{\psi}}{P_{\Omega}}=\frac{\int_{0}^{u_{0}} P(u) \mathrm{d} u}{\int_{0}^{1} P(u) \mathrm{d} u} .
$$

Using the BSO algorithm to solve the optimization problem, the corresponding optimal element distribution is summarized in Table 1 (all the elements are normalized to $0.4 \lambda$, that is, $5.625 * 0.4 \lambda=2.25 \lambda=L)$. As we can see from Table 1 , all antenna elements are within the aperture range, and the spacing of all adjacent antenna elements is less than $d_{c}$.

Table 2 shows the comparison between GA and BSO when $u_{0}$ is different, including the comparison between the maximum value and the average value. It is not difficult to find that both the maximum value and the average value of BSO are better than those of GA. With the increase of $u_{0}$, the average characteristic of BSO is more and more prominent than GA. Moreover, the corresponding power pattern is shown in Figure 3. No matter how many antenna elements there are, the power pattern will always exhibit symmetry property.

Additionally, the best and the average convergence rate curves of the fitness function are plotted in Figure 4. It is obvious that although the convergence rate of BSO is slower than that of GA, the BCE optimized by BSO is higher than that optimized by GA.

The other set of experiments provided the case of $u_{0}=0.2,2 N=10,20$, and $2 N+1=15$ elements; the aperture size is constrained by $2 L=4.5 \lambda, 9.5 \lambda$, and $7 \lambda$, respectively. Also, we changed the size of the receiving region $\psi$ by changing $u_{0}$. In this case, the BCE also can be formulated the same as (10). The conditions are kept the same as the first example, for $2 N=10,20$ elements, $l_{0}$ is chosen as $l_{0}=0.5 d_{c}$, 


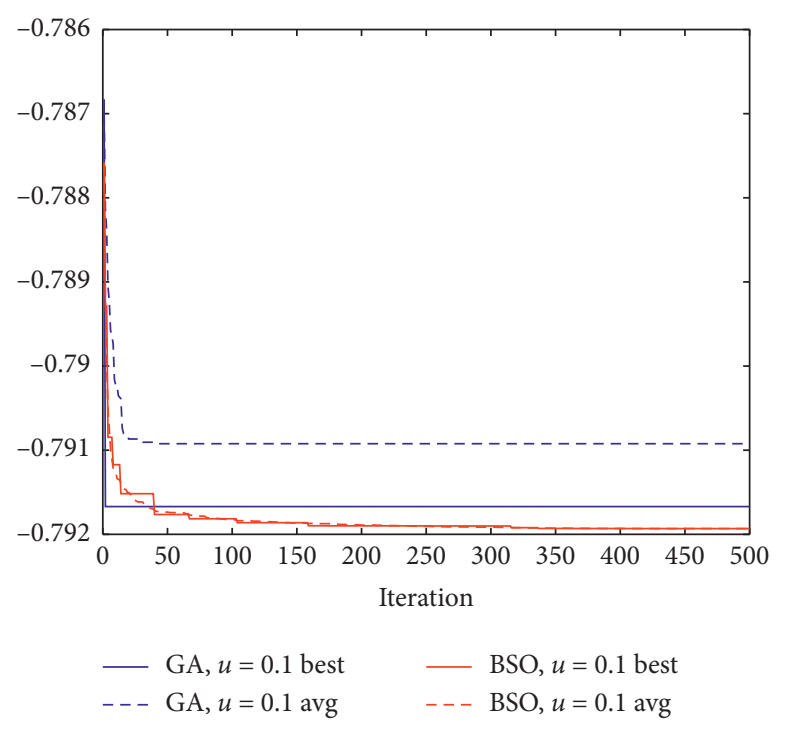

(a)

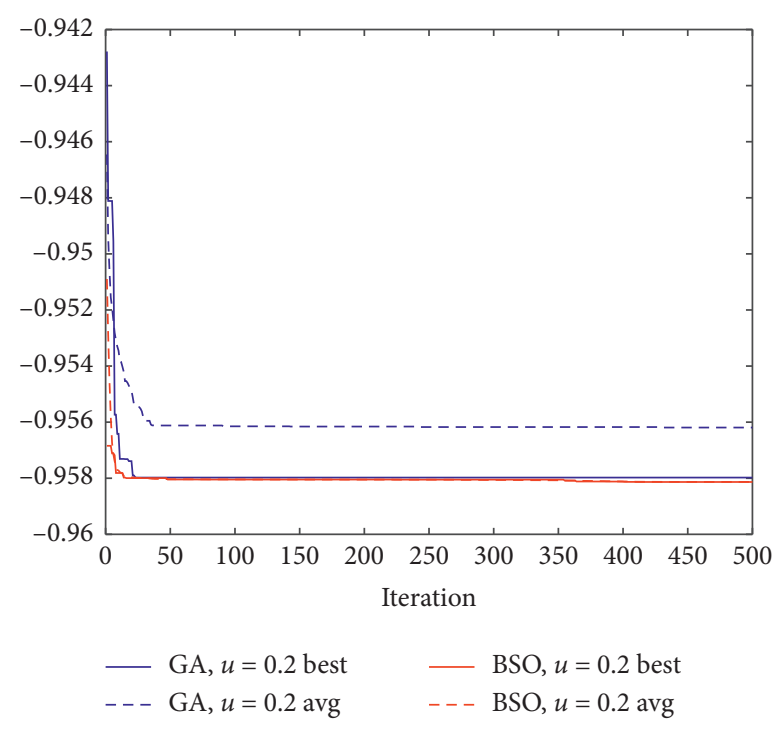

(b)

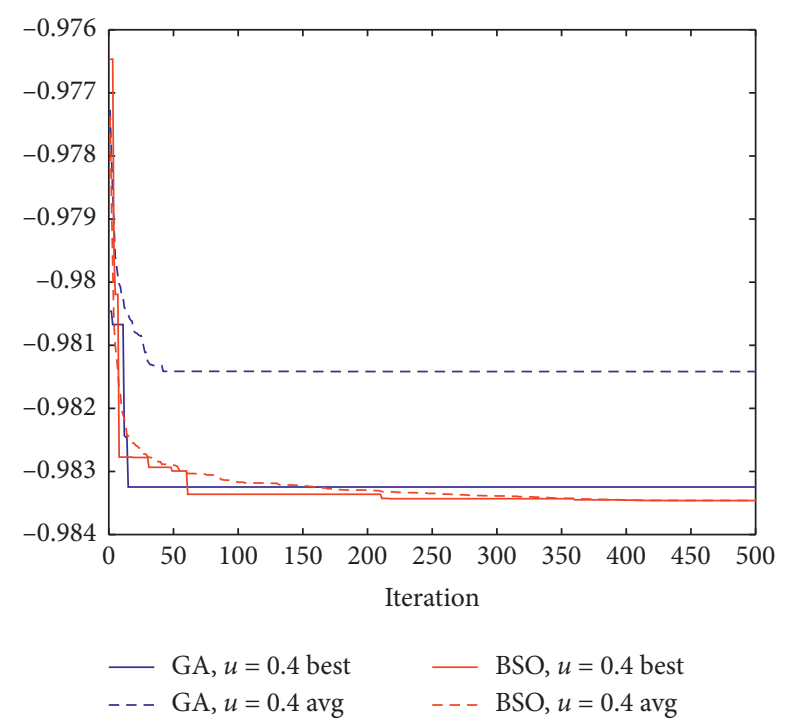

(c)

FIgURE 4: Comparative study on fitness: (a) $u=0.1$; (b) $u=0.2$; (c) $u=0.4$.

whereas, for $2 N+1=15$ elements, $l_{0}$ is chosen as $l_{0}=d_{c}$. In the same way, the corresponding optimal element distribution is summarized in Table 3 (all the elements are normalized to $0.4 \lambda$ ). The same result can be concluded.

According to the number of elements, the comparison between BSO and GA is shown in Table 4. Similarly, the comparisons between the maximum value and the average value are included. Moreover, the optimum power pattern and the convergence rate curves of the fitness function are plotted in Figures 5 and 6, respectively, and similar conclusions can be drawn. The abovementioned numerical results demonstrate that the BSO runs well with great efficiency and considerable stability.

All the experiments were simulated on MATLAB 2018a, and the average time of each simulation was about $30 \mathrm{~s}$. The configuration of the computer is Intel Core i7-7700 at $3.6 \mathrm{GHz}$ with $8 \mathrm{~GB}$ RAM.
TABLE 3: Maximum solutions in BSO, $u_{0}=0.2$, normalized to $0.4 \lambda$.

\begin{tabular}{llccccc}
\hline $2 N(+1)$ & & \multicolumn{5}{c}{ Optimized $d$ vector } \\
\hline 10 & $d=$ & $(0.517$ & 1.517 & 2.613 & 3.945 & $5.625)$ \\
15 & $d=$ & $(1.000$ & 2.048 & 3.048 & 4.231 & 5.359 \\
& & 6.963 & $8.750)$ & & & \\
20 & $d=$ & $(0.756$ & 2.221 & 3.474 & 4.674 & 5.674 \\
& & 6.777 & 7.777 & 8.926 & 10.226 & $11.875)$ \\
\hline
\end{tabular}

TABLE 4: Maximum and average BCE in BSO and GA.

\begin{tabular}{lcccccc}
\hline $2 N(+1)$ & \multicolumn{2}{c}{10} & \multicolumn{2}{c}{15} & \multicolumn{2}{c}{20} \\
BCE (\%) & Max. & Avg. & Max. & Avg. & Max. & Avg. \\
\hline GA & 95.80 & 95.62 & 96.46 & 95.87 & 97.62 & 97.26 \\
BSO & $\mathbf{9 5 . 8 1}$ & $\mathbf{9 5 . 8 1}$ & $\mathbf{9 6 . 6 9}$ & $\mathbf{9 6 . 6 9}$ & $\mathbf{9 7 . 8 4}$ & $\mathbf{9 7 . 8 0}$ \\
Comparison & 0.01 & 0.19 & 0.25 & 0.82 & 0.22 & 0.54 \\
\hline
\end{tabular}




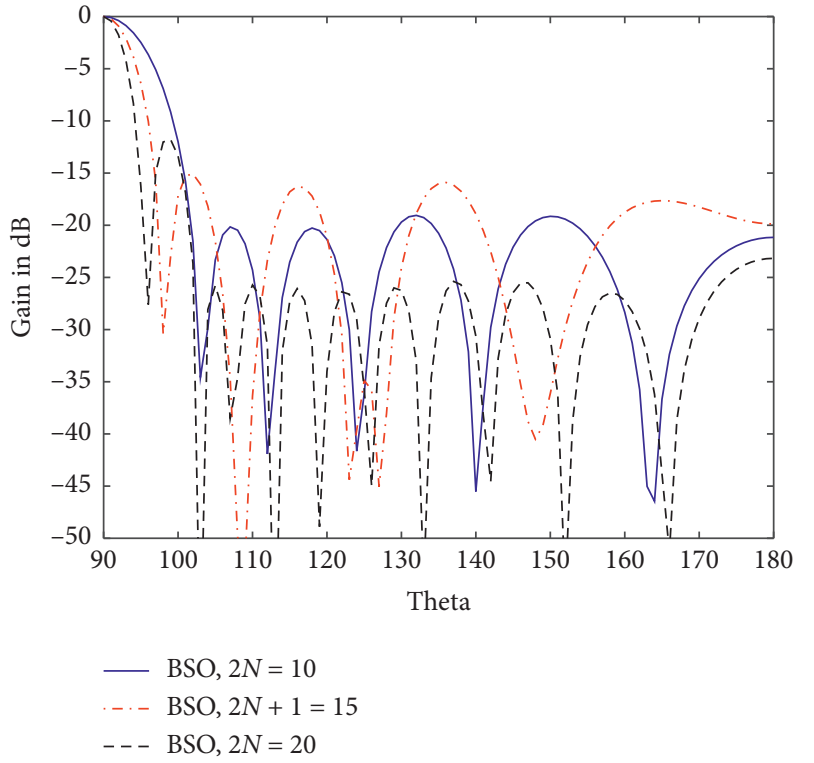

(a)

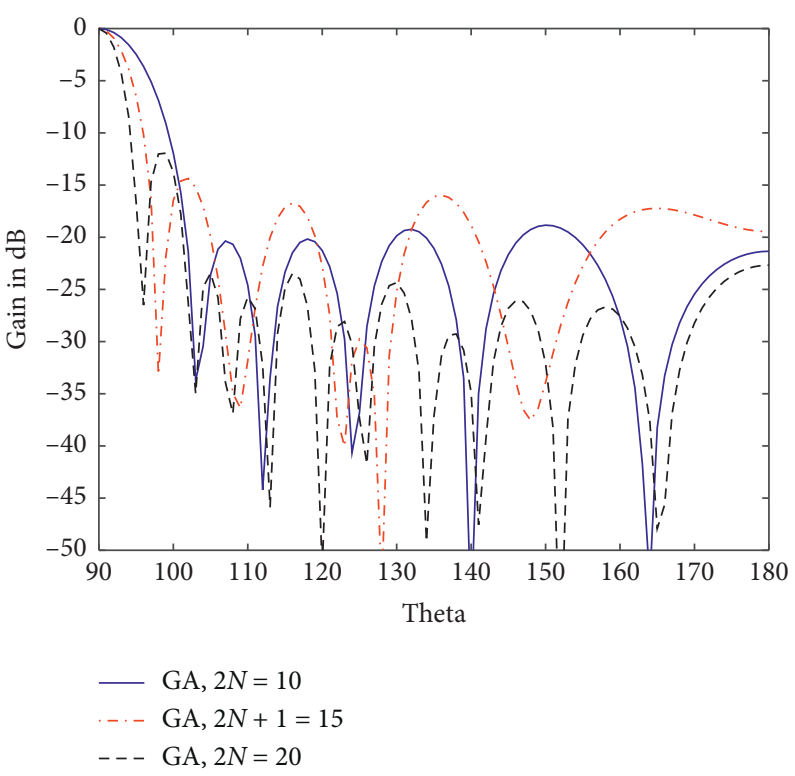

(b)

FIGURE 5: Different element examples: best-normalized power pattern found by BSO (a) and GA (b) (in 20 times runs).

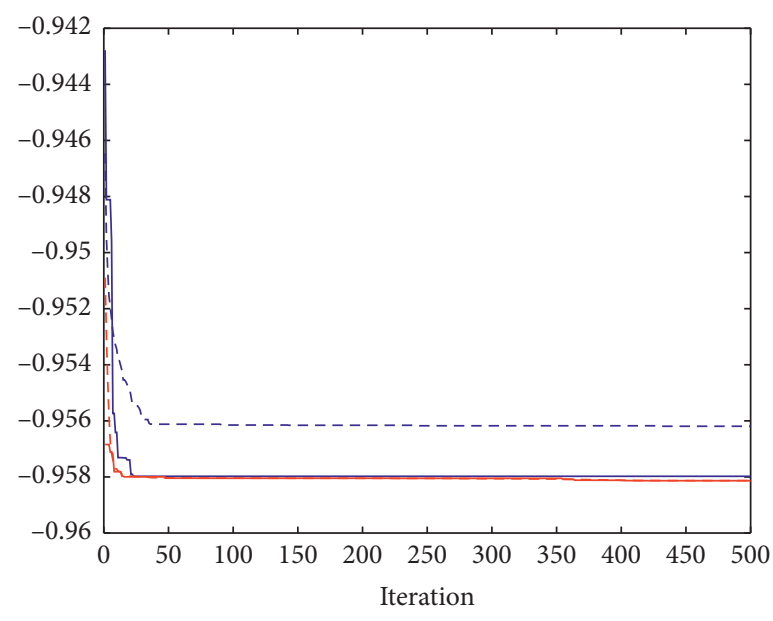

$-\mathrm{GA}, 2 N=10$, best $\quad$ BSO, $2 N=10$, best
$--\mathrm{GA}, 2 N=10$, avg $\quad--$ BSO, $2 N=10$, avg

(a)

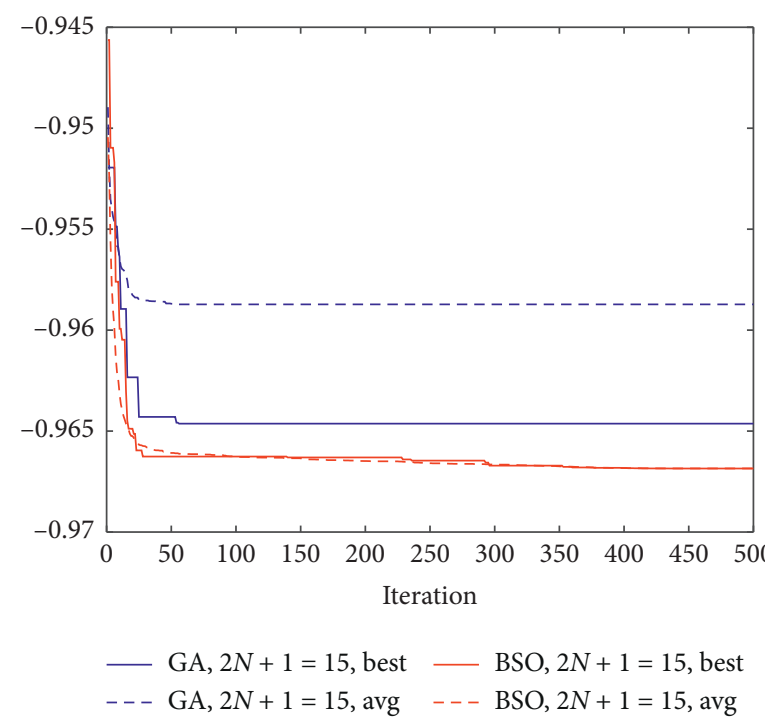

(b)

FIgURE 6: Continued. 


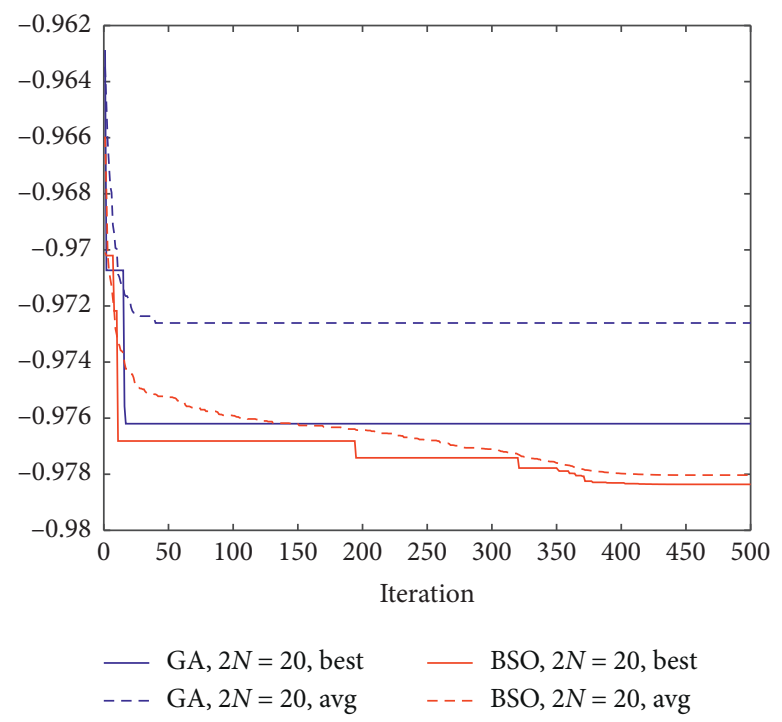

(c)

Figure 6: Comparative study on fitness: (a) $2 N=10$; (b) $2 N+1=15$; (c) $2 N=20$.

\section{Conclusion}

Based on BSO algorithm and taking BCE as objective function, this paper studies the optimization problem under different aperture, different number of antenna elements, and different antenna angles. All experiments show that BSO can better deal with the maximum objective function problem. In this paper, we use BSO algorithm to improve $\mathrm{BCE}$ and prove that $\mathrm{BSO}$ algorithm is more practical and reliable than GA algorithm. However, this paper also has some shortcomings, such as the algorithm used, which is relatively primitive, and only the one-dimensional antenna array is studied. Therefore, in the future, we will study the application of other improved algorithms in this field and try to extend this synthesis technology to more practical antenna structures, such as planar arrays of practical antenna elements and planar antenna power pattern synthesis.

\section{Data Availability}

The data used to support the findings of this study are included within the article.

\section{Conflicts of Interest}

The authors declare that there are no conflicts of interest regarding the publication of this paper.

\section{Acknowledgments}

This work was supported by the National Natural Science Foundation of China under Grant no. 61771008.

\section{References}

[1] W. C. Brown, "The history of power transmission by radio waves," IEEE Transactions on Microwave Theory and Techniques, vol. 32, no. 9, pp. 1230-1242, 1984.
[2] Y.-J. Ren and K. Chang, "New $5.8 \mathrm{GHz}$ circularly polarized retro-directive rectenna arrays for wireless power transmission," IEEE Transactions on Microwave Theory and Techniques, vol. 54, no. 7, pp. 2970-2976, 2006.

[3] H. Oman, "Electric car progress," IEEE Aerospace and Electronic Systems Magazine, vol. 17, no. 6, pp. 30-35, 2002.

[4] J. O. McSpadden and J. C. Mankins, "Space solar power programs and microwave wireless power transmission technology," IEEE Microwave Magazine, vol. 3, no. 4, pp. 46-57, 2002.

[5] A. K. M. Baki, K. Hashimoto, N. Shinohara, T. Mitani, and H. Matsumoto, "New and improved method of beam forming with reduced side lobe levels for microwave power transmission," in Proceedings of the 5th International Conference on Electrical and Computer Engineering, pp. 773-777, Dhaka, Bangladesh, December 2008.

[6] V. Jamnejad and A. Hoorfar, "Optimization of antenna beam transmission efficiency," in Proceedings of the. IEEE Antennas and Propagation Society International Symposium, pp. 1-4, San Diego, CA, USA, July 2008.

[7] G. Oliveri, L. Poli, and A. Massa, "Maximum efficiency beam synthesis of radiating planar arrays for wireless power transmission," IEEE Transactions on Antennas and Propagation, vol. 61, no. 5, pp. 2490-2499, 2013.

[8] X. Li, B. Duan, J. Zhou, L. Song, and Y. Zhang, "Planar array synthesis for optimal microwave power transmission with multiple constraints," IEEE Antennas and Wireless Propagation Letters, vol. 16, pp. 70-73, 2017.

[9] Y. Shi, "Brain storm optimization algorithm," in Advances in Swarm Intelligence, Y. Tan, Y. Shi, Y. Chai, and G. Wang, Eds., pp. 303-309, Springer Berlin Heidelberg, Berlin, Heidelberg, 2011.

[10] A. Aldhafeeri and Y. Rahmat-Samii, "Brain storm optimization for electromagnetic applications: continuous and discrete," IEEE Transactions on Antennas and Propagation, vol. 67, no. 4, pp. 2710-2722, 2019.

[11] R. Salgotra and U. Singh, "The naked mole-rat algorithm," Neural Computing and Applications, vol. 31, no. 12, pp. 8837-8857, 2019. 
[12] R. Salgotra, U. Singh, and S. Saha, "On some improved versions of whale optimization algorithm," Arabian Journal for Science and Engineering, vol. 44, no. 11, pp. 9653-9691, 2019.

[13] U. Singh, R. Salgotra, and M. Rattan, “A novel binary spider monkey optimization algorithm for thinning of concentric circular antenna arrays," IETE Journal of Research, vol. 62, no. 6, pp. 736-744, 2016.

[14] U. Singh and R. Salgotra, "Pattern synthesis of linear antenna arrays using enhanced flower pollination algorithm," International Journal of Antennas and Propagation, vol. 2017, Article ID 7158752, 11 pages, 2017.

[15] K. Chen, Z. He, and C. Han, "A modified real GA for the sparse linear array synthesis with multiple constraints," IEEE Transactions on Antennas and Propagation, vol. 54, no. 7, pp. $2169-2173,2006$ 